\title{
Propagation of vibrational modes in classical harmonic lattice with correlated disorder
}

\section{LEONADE D. DA SILVA ${ }^{1}$, ADHEMAR RANCIARO NETO ${ }^{2}$, MESSIAS O. SALES ${ }^{3}$, JOSÉ L.L. DOS SANTOS ${ }^{4}$ and FRANCISCO A.B.F. DE MOURA ${ }^{1}$}

\author{
${ }^{1}$ Instituto de Física, Universidade Federal de Alagoas, 57072-970 Maceió, AL, Brazil \\ ${ }^{2}$ Faculdade de Economia, Administração e Contabilidade, 57072-970 Maceió, AL, Brazil \\ ${ }^{3}$ Instituto Federal do Maranhão/IFMA, Campus São João dos Patos, Rua Padre Santiago, \\ s/n, Centro, 65665-000 São João dos Patos, MA, Brazil \\ ${ }^{4}$ Escola de Educação Básica e Profissional Fundação Bradesco, Avenida Presidente Vargas, \\ s/n, Serraria, 57046-140 Maceió, AL, Brazil \\ Manuscript received on February 12, 2018; accepted for publication on February 14, 2019
}

\begin{abstract}
How to cite: SILVA LD, RANCIARO NETO A, SALES MO, SANTOS JLL AND MOURA FABF. 2019. Propagation of vibrational modes in classical harmonic lattice with correlated disorder. An Acad Bras Cienc 91: e20180114. DOI 10.1590/10.1590/0001-3765201920180114.
\end{abstract}

\begin{abstract}
The vibrational modes with nonzero frequency are localized in harmonic lattice with disordered masses. In our work, we investigated numerically the propagation of vibrational energy in harmonic lattice with long-range correlated disordered masses, which are randomly distributed with power law spectrum $\mathrm{S}(\mathrm{k}) \propto \mathrm{k}^{-\alpha}$. For $\alpha=0$, a standard uncorrelated disordered mass distribution was observed and for $\alpha>0$ its distribution exhibits intrinsic long-range correlations. Our procedure was done by the numerical solution of the classical equations for the mass displacement and velocities. Energy flow was investigated after injection of an initial wave-packet with energy $\mathrm{E}_{0}$ and the dynamics of the vibrational energy wave-packet was analyzed. We also investigated the dynamics of a pulse pumped at one side of the lattice. Our calculations suggest that vibrational modes with nonzero frequency propagate within harmonic lattice with correlated disordered masses distribution.
\end{abstract}

Key words: localization, correlated disorder, Anderson localization, harmonic lattice.

\section{INTRODUCTION}

The propagation of general particles in disordered systems represents an interesting issue with several connections with solid state physics, acoustics, electrodynamics, biological systems and other branches of science. Within the solid state physics, the most famous contribution in this area is Anderson's work (Anderson 1958). In 1958, P.W. Anderson investigated electron wave-function in disordered theoretical samples. The model consists of a one-electron with local kinetic energy moving in a disordered potential. The random potential simulates the local interaction between the electron and the atoms along an amorphous

Correspondence to: Francisco Anacleto Barros Fidelis de Moura

E-mail: fidelis@fis.ufal.br

ORCid: https://orcid.org/0000-0002-4446-0450 
material. Anderson localization theory demonstrated that, in low-dimensional systems $(d \leq 2)$, the electronic dynamics is absent for any amount of disorder (Kramer and MacKinnon 1993, Abrahams et al. 1979). The results of the Localization Theory not only had a great impact in the physics of electronics systems. but also had thorough influence in acoustic waves (Weaver and Burkhardt 1994), electromagnetic waves (John 1984), light propagation (Störzer 2006), photonic bandgap materials (Schwartz et al. 2007), cold atoms (Izrailev et al. 2012), etc.

The theoretical approach of Anderson takes into consideration the "uncorrelated" disorder distribution within those systems. The term "uncorrelated disorder" represents a class of probability distribution in which intrinsic correlation between two distinct sites is zero. It was shown, years ago, that the Anderson localization predictions in low-dimensional disordered systems may be violated in the case of special kinds correlated disorder (Flores 1989, Dunlap et al. 1990, Bellani et al. 1999, de Moura and Lyra 1998, Domínguez-Adame et al. 2003, de Moura 2010, Izrailev and Krokhin 1999, Izrailev et al. 2001, Kuhl et al. 2000, 2008, Croy and Schreiber 2012, Albrecht and Wimberger 2012). This phenomenon can be achieved by implementing several operations. One of the most famous and successful ways to obtain extended electronic states in disordered chains is by means of a long-range correlated disorder. In general, a disorder distribution with long-range correlations does not exhibit an intrinsic correlation length. The correlation functions exhibit roughly a power law decay (Izrailev et al. 2012, de Moura and Lyra 1998).

The main results of the localization theory can also be applied within the context of vibrational modes in disordered harmonic systems. Vibrational modes with high frequencies in one-dimensional (1D) harmonic chains with a random sequence of masses are localized (Dean 1964). Moreover, there are a few low-frequency modes not localized, whose number is of the order of $\sqrt{\mathrm{N}}$, where $\mathrm{N}$ is the number of masses in the chain (Dean 1964, Matsuda and Ishii 1970, Ishii 1973). The effect of correlated disorder in classical one-dimensional harmonic chains was properly investigated. In harmonic chains with correlated disorder, appearance of new non-scattered vibrational modes were detected (Datta and Kundu 1994, de Moura et al. 2003, Albuquerque 2005). Other dimensionality, such as $d=2$, still deserves a more intense attention, in special, at the presence of correlated disorder.

Despite the past analysis of two-dimensional harmonic lattice with long-range correlation (de Moura and Domínguez-Adame 2008), authors did not account for transversal atomic displacements in their model. They found numerical proof that extended longitudinal modes can appear for strong correlations.

In this work, we considered the problem of a harmonic 2D lattice with correlated disorder and masses' movements in transversal and longitudinal ways (i.e., $\mathrm{x}$ and $\mathrm{y}$ directions). In our model, the random masses distribution exhibits a power-law spectral density $S(k) \sim 1 / \mathrm{k}^{\alpha}$. For $\alpha=0$ we recovered the standard disordered harmonic lattice. For $\alpha>0$, the masses distribution contains long-range correlations that decays approximately as a power law. We solved the classical equations using a second order Euler formalism. Our numerical experiments consists of energy injection into the lattice and track the evolution of the wave-packet. Our calculations suggest that intrinsic correlations, which exists within the masses' distribution, promotes a ballistic energy flux throughout those disordered systems.

\section{MODEL AND NUMERICAL CALCULATIONS}

We consider a two-dimensional harmonic lattice $(\mathrm{L} \times \mathrm{N})$ where each site $(\mathrm{n}, \mathrm{m})$ represents an atom with mass $\mathrm{M}_{\mathrm{n}, \mathrm{m}}$. When the system is at rest, the $\mathrm{x}$ and $\mathrm{y}$ coordinates may be set to $\mathrm{x}_{\mathrm{n}, \mathrm{m}}=\mathrm{m}$ and $\mathrm{y}_{\mathrm{n}, \mathrm{m}}=\mathrm{n}$ (in 

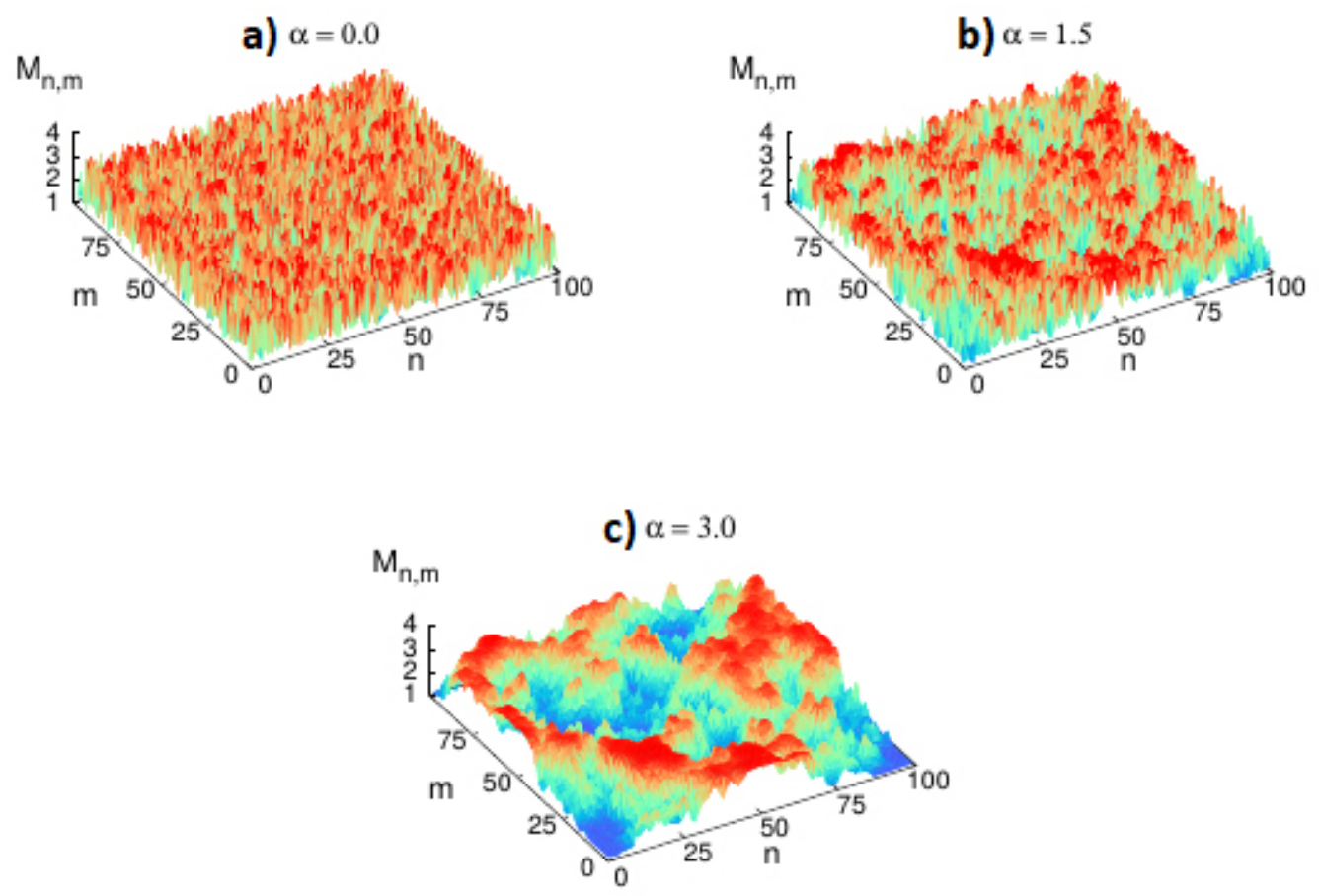

Figure $1-M_{n, m} \times n \times m$ for $L=N=100$ and $\left.\left.\alpha=a\right) 0, b\right) 1.5$ and c)3. We observe that as $\alpha$ is increased, masses' distribution becomes smoother due to the presence of long-range correlations.

$$
\text { a) } \alpha=0.0
$$

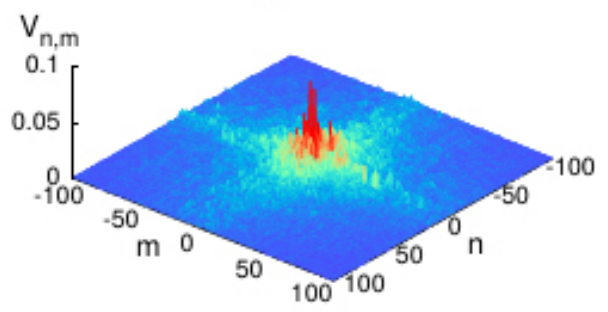

b) $\alpha=3.0$

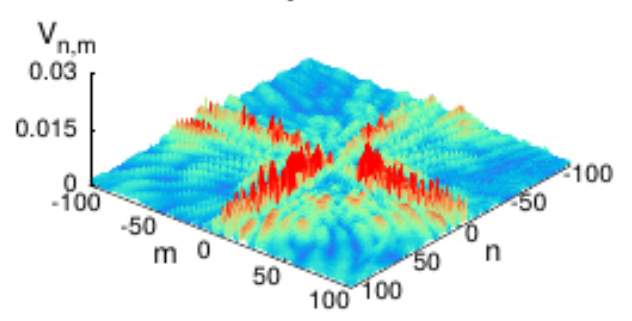

Figure 2 - $(\mathbf{a}, \mathbf{b})$ Velocity of mass $n, m\left(V_{n, m}=\sqrt{\left(v_{n, m}^{x}\right)^{2}+\left(v_{n, m}^{y}\right)^{2}}\right)$ versus $n$ and $m$ for $\alpha=0$ and $\alpha=3$. Calculations were done in a square lattice with $\mathrm{N} \times \mathrm{N}=1500 \times 1500(\mathrm{n}=\mathrm{m}=0$ represent the center of lattice). Classical equations were integrated until $\mathrm{t}_{\max } \approx 600$. We observe clearly in $(\mathrm{b})$ the effect of intrinsic correlations promoting a fast and intense vibrational energy propagation. 

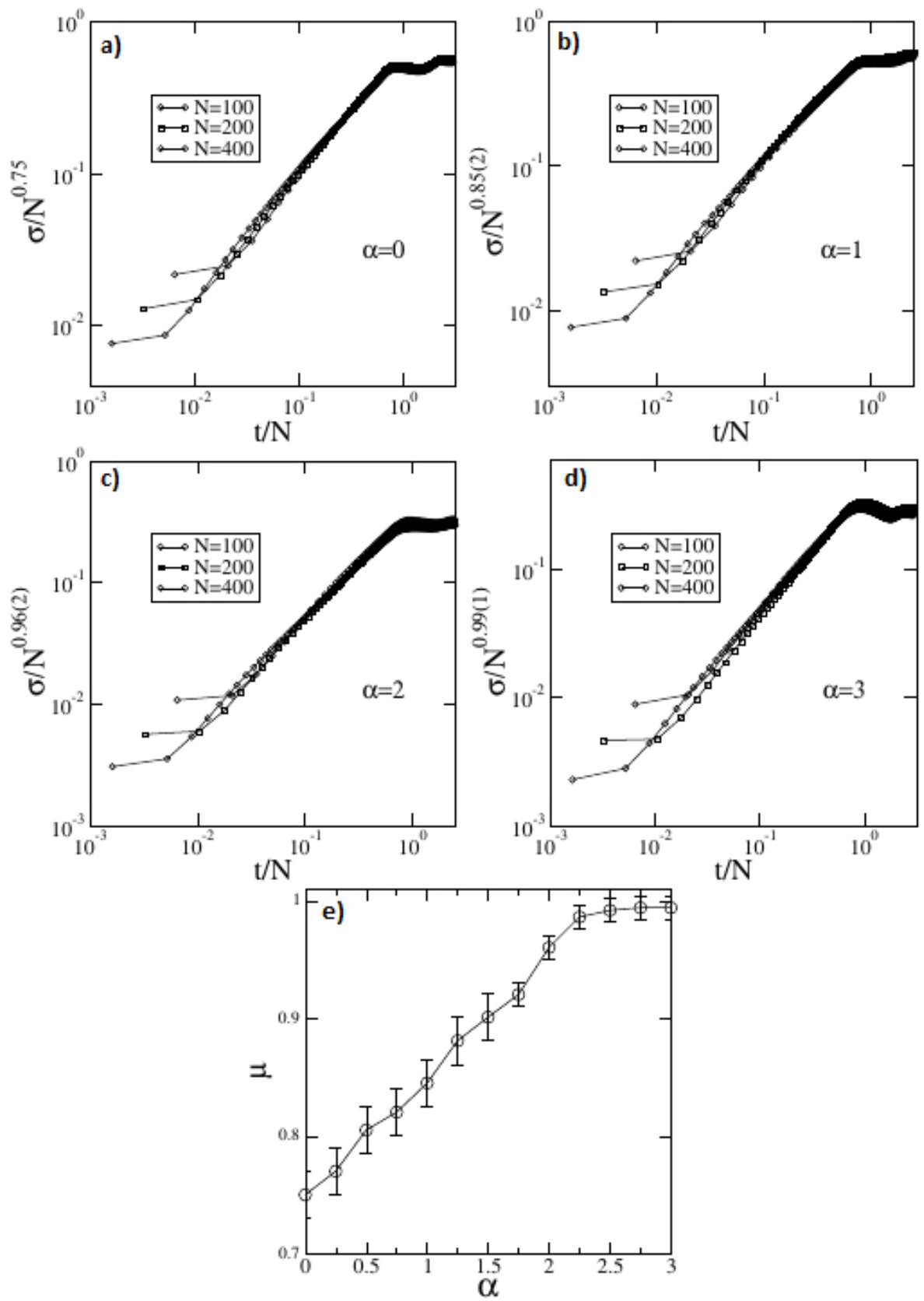

Figure 3 - (a-d) Rescaled energy spread $\sigma(\mathrm{t}) / \mathrm{N}^{\mu}$ versus rescaled time $\mathrm{t} / \mathrm{N}$ for $\alpha=$ (a) 0 , (b) 1 , (c) 2 and (d) 3. The initial condition used was $\mathrm{x}_{\mathrm{n}, \mathrm{m}}(\mathrm{t}=0)=\mathrm{m}+\Delta \delta_{\mathrm{n}, \mathrm{n}_{0}} \delta_{\mathrm{m}, \mathrm{m}_{0}}, \mathrm{y}_{\mathrm{n}, \mathrm{m}}(\mathrm{t}=0)=\mathrm{n}+\Delta \delta_{\mathrm{n}, \mathrm{n}_{0}} \delta_{\mathrm{m}, \mathrm{m}_{0}}$ and $\mathrm{v}_{\mathrm{x}}^{\mathrm{n}, \mathrm{m}}(\mathrm{t}=0)=\mathrm{v}_{\mathrm{y}}^{\mathrm{n}, \mathrm{m}}(\mathrm{t}=0)=0$ (here we used $\left.\Delta=0.5\right)$. The data collapse for $\alpha=3$ suggests occurrence of ballistic dynamics $\left(\sigma(\mathrm{t}) \propto \mathrm{t}^{1}\right)$. For $\alpha<2$, our calculations point to a superdifusive dynamics with exponent $\varsigma \propto t^{\mu}$ with $\mu<1$. In (e) we plot the evolution of the exponent $\mu$ as the correlation becomes stronger. 

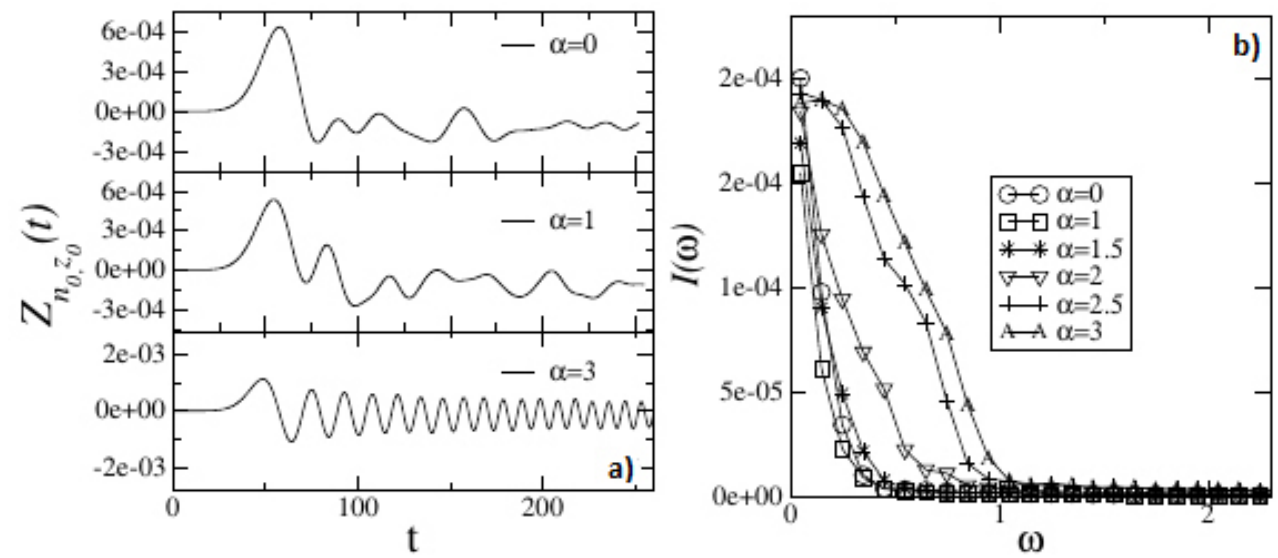

Figure 4 - a) Calculations of the energy flux through the harmonic lattice with correlated disorder. We plot $\mathrm{Z}_{\mathrm{n}_{0}, \mathrm{z}_{0}}$ versus $\mathrm{t}$ for $\mathrm{N}=2400, \mathrm{~L}=360, \mathrm{n}_{0}=\mathrm{L} / 2, \mathrm{z}_{0}=1300$ and $\alpha=0,1$ and 3 . b)I $(\omega)=\left|\mathrm{FT}\left(\mathrm{Z}_{\mathrm{n}_{0}, \mathrm{z}_{0}}(\mathrm{t})\right)\right|$ versus $\omega$ for several values of $\alpha$. For $\alpha>2$, our calculations suggest the existence of a phase of extended vibrational modes with frequencies within the interval $\left[0, \omega_{c}\right]$.

units of the lattice spacing $\mathrm{a}=1$ ), respectively. Masses are randomly distributed with power law spectrum $\mathrm{S}(\mathrm{k}) \propto \mathrm{k}^{-\alpha}$. For $\alpha=0$ we get an uncorrelated disordered mass distribution. For $\alpha>0$, mass distribution exhibits intrinsic long-range correlations. In order to generate the long-range correlated mass distribution, we used the framework similar to those presented in refs. (dos Santos et al. 2007), applying the formula $\Theta_{\mathrm{n}, \mathrm{m}}$ defined as:

$$
\begin{array}{r}
\Theta_{\mathrm{n}, \mathrm{m}}=\sum_{\mathrm{k}_{\mathrm{n}}=1}^{\mathrm{L} / 2} \sum_{\mathrm{k}_{\mathrm{m}}=1}^{\mathrm{N} / 2} \frac{\zeta(\alpha)}{\left(\mathrm{k}_{\mathrm{n}}^{2}+\mathrm{k}_{\mathrm{m}}^{2}\right)^{\alpha / 4}} \times \\
\cos \left(\frac{2 \pi \mathrm{nk} \mathrm{n}_{\mathrm{n}}}{\mathrm{L}}+\psi_{\mathrm{i}, \mathrm{m}}\right) \cos \left(\frac{2 \pi m k_{m}}{\mathrm{~N}}+\varphi_{\mathrm{i}, \mathrm{m}}\right),
\end{array}
$$

where $\psi_{n, m}$ and $\varphi_{n, m}$ are independent random phases uniformly distributed in the interval $[0,2 \pi]$ and $\zeta(\alpha)$ is a normalization constant. We normalized $\Theta_{\mathrm{n}, \mathrm{m}}$ in such a way that $\left\langle\Theta_{\mathrm{n}, \mathrm{m}}>=0\right.$ and $\left\langle\left(\Theta_{\mathrm{n}, \mathrm{m}}\right)^{2}=\right.$ $1>$. The mass $M_{n, m}$ of our harmonic system is defined as $M_{n, m}=\tanh \left(\Theta_{n, m}\right)+2$. We emphasize that this transformation of $\Theta_{n, m}$ using hiperbolic function did not change the power law spectrum $\left(S(k) \propto k^{-\alpha}\right)$. This transformation generated a disorder distribution $\mathbf{M}_{n, m}$ with the same intrinsic correlations as in $\Theta_{n, m}$ within the interval $[1,3]$.

The distance $d_{1}^{n, m}$ between the site $(n, m)$ and its four first nearest neighbors $(1=1,2,3,4)$ after a spring deformation around site $\mathrm{n}, \mathrm{m}$ was measured as:

$$
\begin{aligned}
& d_{1}^{n, m}=\sqrt{\left(x_{n, m}-x_{n+1, m}\right)^{2}+\left(y_{n, m}-y_{n+1, m}\right)^{2}}, \\
& d_{2}^{n, m}=\sqrt{\left(x_{n, m}-x_{n, m+1}\right)^{2}+\left(y_{n, m}-y_{n, m+1}\right)^{2}}, \\
& d_{3}^{n, m}=\sqrt{\left(x_{n, m}-x_{n-1, m}\right)^{2}+\left(y_{n, m}-y_{n-1, m}\right)^{2}}
\end{aligned}
$$


and

$$
\mathrm{d}_{4}^{\mathrm{n}, \mathrm{m}}=\sqrt{\left(\mathrm{x}_{\mathrm{n}, \mathrm{m}}-\mathrm{x}_{\mathrm{n}, \mathrm{m}-1}\right)^{2}+\left(\mathrm{y}_{\mathrm{n}, \mathrm{m}}-\mathrm{y}_{\mathrm{n}, \mathrm{m}-1}\right)^{2}} .
$$

In this model, the effective force on mass $\left(\mathrm{M}_{\mathrm{n}, \mathrm{m}}\right)$ was computed as $\overrightarrow{\mathrm{F}}^{\mathrm{n}, \mathrm{m}}=\overrightarrow{\mathrm{F}}_{1}^{\mathrm{n}, \mathrm{m}}+\overrightarrow{\mathrm{F}}_{2}^{\mathrm{n}, \mathrm{m}}+\overrightarrow{\mathrm{F}}_{3}^{\mathrm{n}, \mathrm{m}}+\overrightarrow{\mathrm{F}}_{4}^{\mathrm{n}, \mathrm{m}}$ where $\overrightarrow{\mathrm{F}}_{1}^{\mathrm{n}, \mathrm{m}}=\mathrm{Kld} \mathrm{n}_{1}^{\mathrm{n}, \mathrm{m}}-1 \mid \hat{\mathrm{r}}_{1}$ with $\mathrm{l}=1,2,3,4$. $\mathrm{K}$ is value of the spring constant and $\hat{\mathrm{r}}_{1}$ is an unity vector along the direction $1(\mathrm{~K}=1$ here $)$. We stress that the direction of $\hat{\mathrm{r}}_{1}$ depends on the distance $\mathrm{d}_{1}^{\mathrm{n}, \mathrm{m}}:$ if $\mathrm{d}_{1}^{\mathrm{n}, \mathrm{m}}>1$, then the vector $\hat{r}_{1}$ points to the direction of mass $n, m$; on the other hand, if $d_{1}^{n, m}<1$, then $\hat{r}_{1}$ points to the opposite direction of mass $\mathrm{n}, \mathrm{m}$. The vibrational energy dynamic process is obtained by solving the classical equations:

$$
\left[\overrightarrow{\mathrm{F}}^{\mathrm{n}, \mathrm{m}}\right]_{\mathrm{x}}=\mathrm{M}_{\mathrm{n}, \mathrm{m}} \frac{\mathrm{d}^{2} \mathrm{x}_{\mathrm{n}, \mathrm{m}}}{\mathrm{dt}^{2}}
$$

and

$$
\left[\overrightarrow{\mathrm{F}}^{\mathrm{n}, \mathrm{m}}\right]_{\mathrm{y}}=\mathrm{M}_{\mathrm{n}, \mathrm{m}} \frac{\mathrm{d}^{2} \mathrm{y}_{\mathrm{n}, \mathrm{m}}}{\mathrm{dt}^{2}}
$$

In general, eqs. 6 and 7 were solved in the following manner: each second order equation was separated into two first order equations (for the eqs. 6 and 7, we get: $\left[\vec{F}^{n, m}\right]_{j}=M_{n, m} \frac{d v_{j}^{n, m}}{d t}$ and $v_{j}^{n, m}=\frac{d j_{n, m}}{d t}$, where $j=$ $\mathrm{x}, \mathrm{y}$, respectively). Therefore, we have a set of four equations for each mass $(\mathrm{n}, \mathrm{m})$ which can be solved using a second order Euler method (2EM). In order to explain the (2EM) method, we used, as example, the two first order equations for the $x$ direction. Assuming that we know the initial value of $x_{n, m}(t=0)$ and $v_{x}^{n, m}(t=0)$, we find a first order estimation for these quantities at time $d t$ as: $x_{n, m}(t=d t)^{1}=x_{n, m}(t=0)+d t * v_{x}^{n, m}(t=0)$ and $v_{x}^{n, m}(t=d t)^{1}=v_{x}^{n, m}(t=0)+d t *\left[\vec{F}^{n, m}(t=0)\right]_{x} / M_{n, m}$. The second order formulae for these quantities is written as: $\mathrm{x}_{\mathrm{n}, \mathrm{m}}(\mathrm{t}=\mathrm{dt})^{2}=\mathrm{x}_{\mathrm{n}, \mathrm{m}}(\mathrm{t}=0)+(\mathrm{dt} / 2) *\left(\mathrm{v}_{\mathrm{x}}^{\mathrm{n}, \mathrm{m}}(\mathrm{t}=0)+\mathrm{v}_{\mathrm{x}}^{\mathrm{n}, \mathrm{m}}(\mathrm{t}=\mathrm{dt})^{1}\right)$ and $\mathrm{v}_{\mathrm{x}}^{\mathrm{n}, \mathrm{m}}(\mathrm{t}=\mathrm{dt})^{2}=\mathrm{v}_{\mathrm{x}}^{\mathrm{n}, \mathrm{m}}(\mathrm{t}=$ $0)+(\mathrm{dt} / 2) *\left(\left[\overrightarrow{\mathrm{F}}^{\mathrm{n}, \mathrm{m}}(\mathrm{t}=0)\right]_{\mathrm{X}}+\left[\overrightarrow{\mathrm{F}}^{\mathrm{n}, \mathrm{m}}(\mathrm{t}=\mathrm{dt})\right]_{\mathrm{X}}\right) / \mathrm{M}_{\mathrm{n}, \mathrm{m}}$. The same second order procedure is employed for the y direction. In our calculations, we used $\mathrm{dt} \approx 5 \times 10^{-3}$ along the entire time interval. We also checked for numerical accuracy of our method. It was done by monitoring the temporal evolution of the total energy contained within the lattice. If an initially localized wave-packet with energy $\mathrm{E}_{0}$ was injected into the lattice, then the time-dependent total energy $\mathrm{E}(\mathrm{t})$ would be constant along the time. We found $\left|1-\mathrm{E}(\mathrm{t}) / \mathrm{E}_{0}\right|<10^{-10}$ within the entire interval.

Our first analysis consists of injecting an initial wave-packet with energy $E_{0}$ at the center $(N / 2, L / 2)$ of a square lattice (i.e., $\mathrm{N}=\mathrm{L}$ ) and then calculating its spread along time. The fraction $\mathrm{R}_{\mathrm{n}, \mathrm{m}}$ of the initial energy on the mass $(n, m)$ was used to estimate the spread of energy within the lattice. $R_{n, m}$ is defined as $\mathrm{R}^{\mathrm{n}, \mathrm{m}}=\mathrm{h}_{\mathrm{n}, \mathrm{m}} / \mathrm{E}_{0}$, where $\mathrm{h}_{\mathrm{n}, \mathrm{m}}$ is the classical hamiltonian at the mass $\mathrm{n}, \mathrm{m}\left(\mathrm{h}_{\mathrm{n}, \mathrm{m}}=\mathrm{M}_{\mathrm{n}, \mathrm{m}}\left[\left(\mathrm{v}_{\mathrm{x}}^{\mathrm{n}, \mathrm{m}}\right)^{2}+\left(\mathrm{v}_{\mathrm{y}}^{\mathrm{n}, \mathrm{m}}\right)^{2}\right] / 2+\right.$ $\left.(1 / 4)\left[\left(1-d_{1}\right)^{2}+\left(1-d_{2}\right)^{2}+\left(1-d_{3}\right)^{2}+\left(1-d_{4}\right)^{2}\right]\right)$. After computing $R_{n, m}$ for each $n, m$, we can determine the spread of the energy distribution characterized as:

$$
\sigma(\mathrm{t})=\sqrt{\sum_{\mathrm{n}, \mathrm{m}}\left[(\mathrm{n}-\mathrm{N} / 2)^{2}+(\mathrm{m}-\mathrm{N} / 2)^{2}\right]\left[\mathrm{R}_{\mathrm{n}, \mathrm{m}}\right]}
$$


The quantity $\varsigma$, for extended states, increases ballistically from 0 until it reaches values near N. In the case of localized states, $\varsigma$ remains finite. This analysis gives us a general measure of the energy spread within the lattice. We also performed a direct measure of the propagation of a mode with frequency $\omega$ along the system employing another numerical experiment, with a new topology and initial condition: i) rectangular geometry $(\mathrm{N} \times \mathrm{L}$ with $\mathrm{N}>\mathrm{L})$; ii) one of the sides of this rectangular lattice is now coupled with some oscillators which inject a pulse defined as :

$$
\mathrm{x}_{\mathrm{n}, \mathrm{m}=0}=\sum_{\omega_{\mathrm{n}}} \mathrm{U} \cos \left(\omega_{\mathrm{n}} \mathrm{t}\right)
$$

where $\mathrm{U}$ is a small amplitude and $\omega_{\mathrm{n}}$ is a set of frequencies within the interval $\left[\omega_{\min }, \omega_{\max }\right]=[0.05,5]$. In this second numerical experiment, we are interested in studying energy propagation along one direction. Therefore we solved the equations in a rectangular geometry in which $\mathrm{N}>$ L represents the propagation axis. In order to analyze the energy propagation along the system, we follow the time-evolution of energy pulse by monitoring the mass position $\mathrm{x}_{\mathrm{n}_{0}, \mathrm{z}_{0}}$. In our calculations, $\mathrm{n}_{0} \approx \mathrm{L} / 2$ and $\mathrm{z}_{0}$ is close to $\mathrm{N} / 2$. Hence, $\mathrm{x}_{\mathrm{n}_{0}, \mathrm{z}_{0}}$ represent the position of a mass far from the lattice side which received the energy pumping. We calculated the displacement of mass $\left(\mathrm{n}_{0}, \mathrm{z}_{0}\right)$ relative to the initial condition i.e. $\mathrm{Z}_{\mathrm{n}_{0}, \mathrm{z}_{0}}(\mathrm{t})=\mathrm{x}_{\mathrm{n}_{0}, \mathrm{z}_{0}}(\mathrm{t})-\mathrm{x}_{\mathrm{n}_{0}, \mathrm{z}_{0}}(\mathrm{t}=0$ ) (in units of the lattice spacing $a=1)$. Then, we can computed $I(\omega)=\left|F T\left(Z_{n_{0}, z_{0}}(t)\right)\right|$, where $F T(A)$ represents the Fourier transform of function A. I $(\omega)$ reveals information about the frequencies that propagate along the chain. When $\mathrm{I}(\omega)=0$, the frequency $\omega$ does not propagate along the lattice. If $\mathrm{I}(\omega)>0$, our results provide a numerical demonstration that vibrational modes with frequency $\omega$ evolves along the lattice from one side to the other. In summary, $I(\omega)>0$ suggests propagation and the existence of extended modes with frequency $\omega$.

\section{RESULTS AND DISCUSSION}

We generated the disorder distribution using the procedure defined in the previous section, i.e. $\mathrm{M}_{\mathrm{n}, \mathrm{m}}=$ $\tanh \left(\Theta_{n, m}\right)+2$, where $\Theta_{n, m}$ is defined in eq. 1. In fig. 1, we plot $M_{n, m} \times n \times m$ for $\left.\left.\alpha=a\right) 0, b\right) 1.5$ and c)3. We observed that, for $\alpha=0$, masses are randomly distributed within the interval [1,3]. As the value of $\alpha$ is increased the aforementioned distribution acquires intrinsic correlations and the disorder profile becomes smoother. This phenomenon was studied in detail in ref. (da Silva et al. 2017). Authors gave numerical proof of the smoothness of local disorder as the correlation parameter $\alpha$ increases. It is a consequence of the Fourier method used to generate the correlated disorder. However, in ref. (da Silva et al. 2017) it was also shown that, for $\alpha \leq 3$, the local disorder does not vanish in finite samples. In figs. 2 and 3, we plot results for the time evolution of a initially localized energy wave-packet in a square harmonic lattice with $\mathrm{N}=\mathrm{L}$.

For $\mathrm{t}=0$, an initial energy input was injected on site $\mathrm{N} / 2, \mathrm{~N} 2$ as $\mathrm{x}_{\mathrm{n}, \mathrm{m}}(\mathrm{t}=0)=\mathrm{m}+\Delta \delta_{\mathrm{n}, \mathrm{N} / 2} \delta_{\mathrm{m}, \mathrm{N} / 2}, \mathrm{y}_{\mathrm{n}, \mathrm{m}}(\mathrm{t}=$ $0)=\mathrm{n}+\Delta \delta_{\mathrm{n}, \mathrm{N} / 2} \delta_{\mathrm{m}, \mathrm{N} / 2}$ and $\mathrm{v}_{\mathrm{x}}^{\mathrm{n}, \mathrm{m}}(\mathrm{t}=0)=\mathrm{v}_{\mathrm{y}}^{\mathrm{n}, \mathrm{m}}(\mathrm{t}=0)=0$. This kind of initial excitation is called displacement excitation. Our calculations were done for $\Delta=0.5$ and $\mathrm{dt}=0.005$. The energy conservation was checked for each integration step. We calculated the total energy of the lattice as $E(t)=\sum_{n, m} h_{n, m}$ and our convergence criterion was $\left|1-\mathrm{E}(\mathrm{t}) / \mathrm{E}_{0}\right|<10^{-10}$. In fig. 2 we plot the velocity of mass $\mathrm{n}, \mathrm{m}\left(\mathrm{V}_{\mathrm{n}, \mathrm{m}}=\sqrt{\left(\mathrm{v}_{\mathrm{n}, \mathrm{m}}^{\mathrm{x}}\right)^{2}+\left(\mathrm{v}_{\mathrm{n}, \mathrm{m}}^{\mathrm{y}}\right)^{2}}\right)$ versus $\mathrm{n}$ and $\mathrm{m}$ for $\alpha=0$ and $\alpha=3$. We studied a square lattice with $\mathrm{N} \times \mathrm{N}=1500 \times 1500$ and final time $\mathrm{t}_{\max } \approx 600$ time units. In fig. $2, \mathrm{n}=\mathrm{m}=0$ represents the center of lattice. Results clearly show that for $\alpha=3$, the initial wave-packet spreads over a larger region and also faster than the case with $\alpha=0$. However, fig. 2 represents only a pedagogical visualization about the vibrational energy propagation within our model. 
Now, we discuss the energy propagation by observing the spread of the energy wave-packet i.e. the quantity ५. We plot in fig. 3 (a-d) the re-scaled mean square displacement $\sigma(\mathrm{t}) / \mathrm{N}^{\mu}$ versus scaled time $\mathrm{t} / \mathrm{N}$ computed in lattices $\mathrm{N} \times \mathrm{N}=100 \times 100,200 \times 200$ and $400 \times 400$. Here, we integrated the classical equations until a stationary state is reached after multiple reflections of the energy vibration on the lattice boundaries. It is worth mentioning that the mean-square displacement saturates at a value $\propto \mathrm{N}^{\mu}$ due to finite size effects. Using the scaling variables $\left(\sigma / \mathrm{N}^{\mu}\right.$ versus $\left.\mathrm{t} / \mathrm{N}\right)$ we detect the presence of extended or localized states by analyzing the existence of a data collapse of all curves. The data collapse using the scaled variables suggests that the dynamics for long time is $\varsigma \propto \mathrm{t}^{\mu}$. We notice that, for $\alpha=3$, our calculations indicated the presence of a data collapse with $\mu \approx 1$. It is a signature of ballistic dynamics and, therefore, extended states (see fig. $3 \mathrm{~d}$ ). For $\alpha<2$, we found data collapse with $\mu<1$ (see fig. 3a, c). It is a clear signature of super-diffusive dynamics. We stress that, in disordered harmonic chains, it was demonstrated that the Anderson Localization Theory works for high frequencies, but modes around $\omega=0$ (the uniform mode) can propagate even for strong disorder (Dean 1964, Datta and Kundu 1994). Also, propagation of the uniform mode promotes a diffusive (or super-diffusive) energy propagation along the chain. Our calculations suggest that in disordered lattices with weak correlations in the disorder distribution (i.e $\alpha<2$ ) the energy propagates in a super-diffusive regime $\left(\varsigma \propto t^{\mu}\right.$ with $\left.0.75<\mu<1\right)$ similar to the $1 d$ disordered case (Dean 1964, Datta and Kundu 1994). In fig. 3e we plot the exponent $\mu$ versus $\alpha$ with $\alpha=0$ up to 3 . We clearly see a transition from a super-diffusive regime $(0.5<\mu<1)$ to a ballistic one $(\mu=1)$ as the correlation degree $\alpha$ is increased. The transition point seems to be around $\alpha=2$. We emphasize that the critical point $\alpha=2$ was also found in electronic $1 \mathrm{~d}$ systems with correlated disorder (Izrailev et al. 2012).

In fig. $4 \mathrm{a}$, we plot $\mathrm{Z}_{\mathrm{n}_{0}, \mathrm{z}_{0}}$ versus $\mathrm{t}$ for $\alpha=0,1$ and 3. Calculations were done in a rectangular $(\mathrm{L} \times \mathrm{N})$ harmonic lattice with $\mathrm{N}=2400$ and $\mathrm{L}=360$ and with $\mathrm{n}_{0} \approx \mathrm{L} / 2$ and $\mathrm{z}_{0} \approx 1300$. We emphasize that, in this figure, $4 \mathrm{a}$ we considered $\mathrm{t}=0$ the time in which that $\left|\mathrm{Z}_{\mathrm{n}_{0}, \mathrm{z}_{0}}\right|>10^{-10}$. By using the time-evolution of $\mathrm{Z}_{\mathrm{n}_{0}, \mathrm{Z}_{0}}$ we calculated the quantity $I(\omega)$ as $I(\omega)=\left|\mathrm{FT}\left(\mathrm{Z}_{\mathrm{n}_{0}, \mathrm{z}_{0}}(\mathrm{t})\right)\right|$, where $\mathrm{FT}\left(\mathrm{Z}_{\mathrm{n}_{0}, \mathrm{z}_{0}}(\mathrm{t})\right)$ represents the Fourier transform of $\mathrm{Z}_{\mathrm{n}_{0}, \mathrm{z}_{0}}(\mathrm{t})$. The results of $\mathrm{I}(\omega)$ for several values of $\alpha$ are found in figure $4 \mathrm{~b}$. We emphasize that the calculations of $\mathrm{I}(\omega)$ were averaged using 50 distinct samples. Our calculations were summarized as follows: The function $\mathrm{I}(\omega)$ for the uncorrelated case $\alpha=0$ is almost null for $\omega>0$ and exhibits a pronounced peak around $\omega=0$. Looking in more detail we can see that there is a peak around $\omega=0$ and narrow region with frequency $\omega \approx 0$ that also exhibits $I(\omega)$ slightly larger than zero. This peak and this small region are related with those modes around the uniform mode $(\omega=0)$ which propagates along the lattice (Dean 1964). We emphasize that the phenomenology around the uniform mode $(\omega=0)$ in disordered harmonic systems was first investigated in disordered harmonic chains. This uniform mode represents a mode without spring deformation, so it has divergent wave-length and, also, it is not affected by the disorder propagating through the system (Dean 1964, Datta and Kundu 1994). In two dimensional disordered harmonic systems, the uniform model can also propagate along the lattice, thus promoting the appearance $\mathrm{I}(\omega \approx 0)>0$. For $\alpha=1$ and 1.5 the results of $\mathrm{I}(\omega)$ are quite similar to that obtained for $\alpha=0$. For $\alpha=2$, we observed a small increasing of the frequency interval in which that $\mathrm{I}(\omega)>0$. We think that these results are in good agreement with our previous calculations about the kind of energy propagation. As the correlation parameter exceeds the value $\alpha=2$, we observe that the region of frequencies in which that $\mathrm{I}(\omega)>0$ increases. For $\alpha=3$ we see a phase of extended frequencies with $\mathrm{I}(\omega)>0$ within the interval $0<\omega<\omega_{\mathrm{c}}$ (based on fig. $4 \mathrm{~b} \omega_{\mathrm{c}} \approx 1$ ). These results are strong indications that the vibrational modes with frequency within the interval $[0,1]$ should be extended. 


\section{CONCLUSIONS}

We investigated the nature of vibrational modes in an harmonic lattice with long-range correlated disorder. In our model, the random masses distribution exhibited a power-law spectral density $S(k) \sim 1 / \mathrm{k}^{\alpha}$. For $\alpha=0$, we recovered the standard disordered harmonic lattice and for $\alpha>0$, the masses distribution contains long-range correlations that decays approximately as a power law. Our numerical experiments consisted of injecting energy into the lattice and follow the evolution of the initially localized wave-packet. Our calculations suggested that as the correlation parameter $\alpha$ is increased, the vibrational energy propagation throughout lattice increases. Our calculations indicate that, for $\alpha>2$, the dynamics becomes ballistic and the model contains a phase of extended vibrational modes.

\section{ACKNOWLEDGMENTS}

This work was partially supported by Conselho Nacional de Desenvolvimento Científico e Tecnológico (CNPq), Coordenação de Aperfeiçoamento de Pessoal de Nível Superior (CAPES), and Financiadora de Inovação e Pesquisa (FINEP) (Federal Brazilian Agencies), CNPq-Rede Nanobioestruturas, as well as FAPEAL (Fundação de Amparo à Pesquisa do Estado de Alagoas).

\section{AUTHORS CONTRIBUTIONS}

L.D. da Silva did all calculations of the figures 1, 2 and 3; J.L.L. dos Santos did the calculations of the figure 4; A. Ranciaro Neto, M.O. Sales and F.A.B.F. de Moura wrote the manuscript.

\section{REFERENCES}

ABRAHAMS E, ANDERSON PW, LICCIARDELLO C AND RAMAKRISHNAN TV. 1979. Scaling theory of localization: Absence of quantum diffusion in two dimensions. Phys Rev Lett 42: 673-676.

ALBRECHT C AND WIMBERGER S. 2012. Induced delocalization by correlation and interaction in the one-dimensional Anderson model. Phys Rev B 85: 045107.

ALBUQUERQUE SS, DE MOURA FABA AND MARCELO LL. 2005. Vibrational modes in harmonic chains with diluted disorder. Phys A 357: 165-172.

ANDERSON PW. 1958. Absence of diffusion in certain random lattices. Phys Rev 109: 1492-1505.

BELLANI V, DIEZ E, HEY R, TONI L, TARRICONE L, PARRAVICINI GB, DOMÍNGUEZ-ADAME F AND GÓMEZ-ALCALÁ R. 1999. Experimental evidence of delocalized states in random dimer superlattices. Phys Rev Lett 82: 2159-2162.

CROY A AND SCHREIBER M. 2012. Correlation-strength-driven anderson metal-insulator transition. Appl Phys Lett, 85: 205147.

DA SILVA LD, RANCIARO NETO A, MESSIAS OS, LYRA ML AND DE MOURA FABF. 2017. Optical absorption and delocalization in a quaternary tight-binding chain with correlated disorder. Phys A 486: 895-900.

DEAN P. 1964. Vibrations of glass-like disordered chains. Proc Phys Soc 84: 727.

DATTA PK AND KUNDU K. 1994. The absence of localization in one-dimensional disordered harmonic chains. J Phys Cond Matt 6: 4465.

DE MOURA FABF AND DOMÍNGUEZ-ADAME F. 2008. Extended modes and energy dynamics in two-dimensional lattices with correlated disorder. Eur Phys J B 66: 165-169.

DE MOURA FABF, COUTINHO-FILHO MD, RAPOSO EP AND LYRA ML. 2003. Delocalization in harmonic chains with long-range correlated random masses. Phys Rev B 68: 012202.

DE MOURA FABF, COUTINHO-FILHO MD, RAPOSO EP AND LYRA ML. 2004. Delocalization and ballistic dynamics in the two-dimensional anderson model with long-range correlated disorder. Eur Lett 66:585-591.

DE MOURA FABF AND LYRA ML. 1998. Delocalization in the 1d anderson model with long-range correlated disorder. Phys Rev Lett 81: 3735-3738. 
DOMÍNGUEZ-ADAME F, MALYSHEV VA, DE MOURA FABF AND LYRA M. 2003. Bloch-like oscillations in a one-dimensional lattice with long-range correlated disorder. Phys Rev Lett 91: 197402.

DUNLAP DH, WU HL AND PHILLIPS PW. 1990. Absence of localization in a random-dimer model. Phys Rev Lett 65: 88-91.

FLORES JC. 1989. Transport in models with correlated diagonal and off-diagonal disorder. J Phys Cond Matt 1: 8471.

ISHII K. 1973. Localization of eigenstates and transport phenomena in the one-dimensional disordered system. Prog Theor Phys Suppl 53: 77-138.

IZRAILEV FM, FELIX M., KROKHIN AA AND MAKAROV N. 2012. Anomalous localization in lowdimensional systems with correlated disorder. Phys Rep 512: 125-254.

IZRAILEV FM AND KROKHIN AA. 1999. Localization and the mobility edge in one-dimensional potentials with correlated disorder. Phys Rev Lett 82: 4062-4065.

IZRAILEV FM, KROKLIN AA AND ULLOA SE. 2001. Mobility edge in aperiodic kronig-penney potentials with correlated disorder: Perturbative approach. Phys Rev B 63: 41102.

JOHN S. 1984. Electromagnetic absorption in a disordered medium near a photon mobility edge. Phys Rev Lett 53: $2169-2172$.

KRAMER B AND MACKINNON A. 1993. Localization: theory and experiment. Rep Prog Phys 56: 1469-1564.

KUHL U, IZRAILEV FM AND KROKHIN A. 2008. Enhancement of localization in one-dimensional random potentials with long-range correlations. Phys Rev 100: 126402.

KUHL U, IZRAILEV FM, KROKLIN AA AND STÖCKMANN HJ. 2000. Experimental observation of the mobility edge in a waveguide with correlated disorder. Appl Phys Lett 77: 633.

MATSUDA H AND ISHII K. 1970. Localization of normal modes and energy transport in the disordered harmonic chain. Prog Theor Phys Suppl 45: 56-86.

SCHWARTZ T, BARTAL G, FISHMAN S AND SEGEV M. 2007. Transport and Anderson localization in disordered two-dimensional photonic lattices. Nat 446: 52-55.

WEAVER RL AND BURKHARDT J. 1994. Weak Andreson localization and enhanced backscatter in reverberation rooms and quantum dots. J Acoust Soc Am 96(5): 3186-3190. 